\title{
1 Non-Invasive Detection of Viral Antibodies Using Oral Flocked
}

\section{Swabs}

3 Running Title: Oral flocked swabs to detect viral antibodies

4 Word Counts: Abstract: 173

Text Body: 3,709

5

6 David J. Speicher ${ }^{1,2,3,4, \# \text {, Kathy Luinstra }}{ }^{2}$, Emma J. Smith ${ }^{5}$, Santina Castriciano ${ }^{6}$, Marek

7 Smieja $\mathbf{a}^{1,2}$

$8 \quad{ }^{1}$ Department of Pathology \& Molecular Medicine, McMaster University, Ontario, Canada

$9 \quad{ }^{2}$ Department of Laboratory Medicine, St. Joseph's Healthcare Hamilton, Ontario, Canada

$10 \quad{ }^{3}$ Menzies Health Institute Queensland, Griffith University, Queensland, Australia

$11{ }^{4}$ M.G. DeGroote Institute for Infectious Disease Research, Department of Biochemistry and

12 Biomedical Sciences, DeGroote School of Medicine, McMaster University, Hamilton, Ontario,

13 Canada

$14{ }^{5}$ Department of Mathematics and Statistics, University of Guelph, Ontario, Canada

$15{ }^{6}$ Copan Italia, Brescia, Italy

17 \#Corresponding author:

25 Keywords: oral flocked swabs, saliva, diagnostics, Cytomegalovirus, Epstein-Barr virus,

26 Varicella-Zoster virus, Measles, Mumps 


\section{Highlights}

28 - Oral flocked swabs are an easy, self-collection method for measuring viral antibodies.

29 - Viral IgG is stable on dried oral flocked swabs for at least two years.

30 - Oral swabs are highly sensitive for CMV, VZV, and EBV IgG.

31 - Oral swabs are potentially useful for surveillance and clinical microbiology. 


\section{Abstract}

33 Salivary antibodies are useful in surveillance and vaccination studies. However, low antibody

34 levels and degradation by endonucleases are problematic. Oral flocked swabs are a potential

35 non-invasive alternative to blood for detecting viral antibodies. Serum and saliva collected from

3650 healthy volunteers were stored at $-80^{\circ} \mathrm{C}$; dried swabs at room temperature. Seroprevalence

37 for Cytomegalovirus (CMV), Varicella-Zoster virus (VZV), Epstein-Barr virus (EBV), Measles

38 and Mumps IgG antibodies were determined using commercial ELISAs and processed on an

39 automated platform. For each antibody, swabs correlated well with saliva. For CMV IgG, VZV

40 IgG, and EBV EBNA-1 IgG and VCA IgG, the swab sensitivities compared to serum were

$4195.8 \%, 96 \%, 92.1 \%$ and $95.5 \%$ respectively. For Measles IgG, swab sensitivity was $84.5 \%$.

42 Mumps IgG displayed poor sensitivity for oral swabs (60.5\%) and saliva (68.2\%). Specificities

43 for IgG antibodies were $100 \%$ for CMV, EBV and Mumps. Specificities for VZV and Measles

44 could not be determined due to seropositive volunteers. As oral flocked swabs correlate well

45 with serum, are easy to self-collect and stable at room temperature further research is warranted. 


\section{1. Introduction}

47 Immunological screening for viral antibodies (primarily $\mathrm{IgG}$ ) in serum to assess past infection or

48 vaccine immunity is routinely performed via commercial enzyme immunoassays (EIA) on closed

49 platforms. Serum is the gold standard for determining immune status but is invasive to collect.

50 Saliva has considerable diagnostic potential: it is non-invasive, abundant, easily collected, and

51 representative of oral and systemic health. Salivary diagnostics is rapidly emerging, especially

52 defining biomarkers for point-of-care testing of infectious diseases (1). Salivary antibodies are

53 primarily secretory $\operatorname{IgA}$ from the salivary glands, while $\operatorname{IgG}$ and $\operatorname{IgM}$ are derived from serum

54 plasma cells and passively diffused into the oral cavity via gingival crevicular fluid $(2,3)$.

55 Salivary $\operatorname{IgG}(\mathrm{sIgG})$ is systemically representative and strongly correlates with serum levels, but

56 loads are approximately 1:800 that of serum $(4,5)$. This is problematic for typical closed testing

57 systems that incorporate a 1:100 dilution step. Despite low levels, salivary antibodies are

58 utilized in the U.S. Food and Drug Administration approved OraQuick ADVANCE ${ }^{\circledR}$ Rapid

59 HIV-1/2 Antibody Test (OraSure Technologies, Inc., USA) and OraQuick ${ }^{\circledR}$ HCV Test (OraSure

60 Technologies, Inc.) (1). However, many commercial assays are cost-prohibitive for resource-

61 limited settings.

63 Saliva collection can be difficult in children and hyposalivators, such as immunocompromised

64 patients. Salivary endonucleases are detrimental, remaining active at $-80^{\circ} \mathrm{C}$, necessitating special

65 handling, or storage in proteolytic stabilizers unsuitable for antibody preservation (6). To

66 overcome these limitations, procedures for viral antibody detection were optimized on an open

67 commercial platform for dried oral flocked swabs, after room temperature storage. The

68 efficiency of oral flocked swabs to detect viral antibodies has yet to be determined. Our method 
69 was initially optimized for Cytomegalovirus (CMV) IgG due to its importance in hematopoietic

70 stem cell, solid organ, and haploidentical transplantations as well as prenatal patients $(7,8)$. We

71 then assessed the procedures' potential application to detect Varicella-Zoster virus (VZV),

72 Epstein-Barr virus (EBV), Measles and Mumps IgG.

\section{2. Materials and Methods}

\subsection{Study Population:}

76 Following Hamilton Integrated Research Ethics Board (HiREB \#14-658) approval and written

77 informed consent, two oral swabs, unstimulated saliva, and blood were collected. Optimisation

78 of pre-analytic and analytic procedures was performed on 10 healthy volunteers with known

79 CMV seropositivity (5 positive, 5 negative), and expanded to 50 healthy volunteers for the

80 diagnostic accuracy study. Laboratory staff (15 males:35 females) from St. Joseph's Healthcare

81 Hamilton with an average age of 43.4 years (range: 18-65 years) voluntarily provided all sample

82 types, except for one who could not produce a saliva sample. Two oral swabs (FLOQSwabs ${ }^{\circledR}$

83 \#520C, Copan Italia S.p.A., Brescia, Italy) were collected consecutively by moistening the

84 flocked swab on the tongue and then rotating between the gums and cheek three to five times.

85 Swabs were then dried for an hour inside a biosafety cabinet and stored inverted in a

86 microcentrifuge tube at room temperature prior to elution and at $-20^{\circ} \mathrm{C}$ following elution. Whilst

87 circadian rhythm was not accounted for as swabs were collected at times convenient for the

88 volunteer, participants were asked to refrain from eating or drinking 60-minutes prior to

89 collection. Cell-free unstimulated saliva was collected by expectorating $2-5 \mathrm{~mL}$ into a sterile

$9050 \mathrm{~mL}$ Falcon tube, centrifuging at 2,800 $\times \mathrm{g}$ for 10-minutes and aspirating the supernatant (9). 
91 Supernatant was aliquoted into $1 \mathrm{~mL}$ portions and stored at $-80 \mathrm{C}$; the cell pellet was discarded.

92 Serum was obtained from a $5 \mathrm{~mL}$ blood collection via venipuncture, allowed to clot, centrifuged

93 at 3,000 $\times \mathrm{g}$ for 10 -minutes, and stored at $-80^{\circ} \mathrm{C}$.

\subsection{Development and Optimisation of pre-analytic and analytic procedures:}

96 The first optimisation to determine the optimal dilution for testing oral swabs was performed

97 with a CMV IgG EIA on the ThunderBolt ${ }^{\circledR}$ ELISA Analyzer; both from Gold Standard

98 Diagnostics (GSDx, Davis, CA, USA). To elute viral antibodies, 250 $\mu \mathrm{L}$ PBS was added to a

99 dried swab head, vortexed for 30-seconds, incubated at room temperature for 10-minutes, and

100 centrifuged at $14,000 \times g$ for one minute, before discarding the swab. Serial dilutions (2-fold

101 serially from neat to 1:16) were prepared in PBS and $100 \mu \mathrm{L}$ tested on the ThunderBolt ${ }^{\circledR}$ ELISA

102 Analyzer as per manufacturer's instructions. Repeated measures one-way ANOVA was

103 performed in R3.5.0 in combination with polynomial contrasts to assess the nature and

104 significance of the relationship between dilutions and optical density (O.D.) values (10).

106 The second optimisation determined the effect of tube shape and elution volume on O.D. values

107 and status. Various volumes of PBS $(150 \mu \mathrm{L}, 200 \mu \mathrm{L}$, and $250 \mu \mathrm{L})$ were added to swabs stored in

108 two shapes of microcentrifuge tubes: 2.0mL flat-bottomed, screw cap tubes (SCT-200-Y,

109 Axygen Scientific, Union City, CA, USA) and 1.5mL conical microcentrifuge tubes (MCT-150-

110 C, Axygen Scientific). The volume of PBS recovered was measured for each. To assess the

111 relationship between O.D. values and both tube shape and volume, a linear mixed effects model

112 was fit. O.D. values were treated as the response and volume added and tube type were 
113 considered as the main effects. As sample manipulations could affect positivity only positive

114 patients were considered.

116 The third optimization determined the effect pelleted buccal cells had on O.D. values and

117 positivity of five salivopositive and five salivonegative samples using three methods: 1 . Pellet,

118 measuring the supernatant O.D. values with undisturbed pelleted buccal cells at the bottom of the

119 tube; 2. Supernatant, measuring the supernatant O.D. values after transfer to a new tube without

120 disturbing the pellet; 3. Resuspended; measuring the O.D. values after complete resuspension of

121 the buccal cells. To determine the relationship between resuspension methods, an initial

122 ANOVA followed by paired testing was used. Further pairwise comparisons between O.D.

123 values of the three methods (pellet, supernatant, and resuspended) were constructed using paired

124 t-tests.

126 The fourth optimisation determined the stability of viral antibodies in dried flocked swabs over

127 time by measuring O.D. values from five salivopositive samples at baseline and after two months

128 stored at room temperature. Paired t-tests were used to compare average O.D. values at both

129 time points.

\section{2.3. Diagnostic Accuracy Study}

132 A cross-sectional diagnostic accuracy study was performed to compare oral flocked swabs and 133 unstimulated saliva versus serum as a reference standard, for the detection of various viral134 specific IgG antibodies. As results were similar between two consecutively collected swabs 
135 (data not shown) swab eluates were pooled to facilitate automated testing of multiple analytes.

136 The assays utilized included: CMV IgG EIA, EBV EBNA IgG EIA, EBV VCA IgG EIA,

137 Measles IgG EIA, and Mumps IgG EIA from GSDx; RIDASCREEN ${ }^{\circledR}$ VZV IgG (K5621),

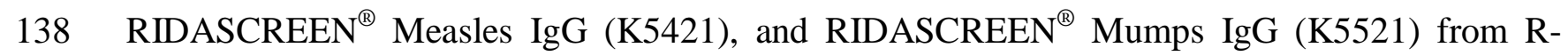

139 Biopharm AG (Darmstadt, Germany). These commercial EIAs are optimised for serum but were

140 used off-label for oral fluids. All assays were performed as per manufacturer's protocol using

$141100 \mu \mathrm{L} 1: 100$ sera, but $100 \mu \mathrm{L}$ undiluted oral fluid. For each assay, the mean O.D. and standard

142 deviation (SD) were calculated from swabs and saliva specimens corresponding to "true-

143 negative" subjects, i.e. serum test negative subjects. We then defined cut-off values as: Non-

144 reactive if less than two SDs above the average O.D. values; Reactive if greater than 3 SDs

145 above the average O.D.; all other O.D. values considered indeterminate. As all samples were

146 seropositive for VZV and measles the cut-off O.D. values for these analytes were extrapolated

147 from the cut-off O.D. values of other assays from the same manufacturer. For each, the

148 diagnostic test accuracy (sensitivity, specificity, positive predictive value (PPV), negative

149 predictive value (NPV), and overall accuracy), and misclassification rates were determined.

150 Kappa statistics were calculated in a pairwise fashion to quantify the agreement beyond chance

151 between oral swabs, unstimulated saliva, and serum for all antibodies of interest.

153 3. Results

\section{3.1. Optimisation of pre-analytic procedures:}

155 To determine the effect of sample dilutions, O.D. values were measured at five, two-fold dilution

156 points. Dilutions significantly decrease O.D. values $(p=0.013)$ and affected positivity: 4-fold and 
157 8-fold dilutions yielded $2 / 5(40 \%)$ and $4 / 5(80 \%)$ false non-reactive samples, respectively

158 (Figure 1). Pairwise comparison showed that dilutions significantly decreased O.D. values in a 159 linear relationship $(\mathrm{F}=17.758, p=0.014)$. Therefore, swabs were used undiluted to ensure that 160 weakly reactive samples did not become falsely non-reactive.

162 To determine the effect of tube shape and establish the optimal elution volume, O.D. values were 163 measured at three volumes of PBS and two tube shape combinations. The conical tube shape 164 caused eluate to be reabsorbed by the swab, significantly reduced recovery volume (conical vs 165 flat-bottomed: $60.66 \pm 4.38 \%$ vs $98.63 \pm 0.78 \% ; p=0.017)$, O.D. values $(p=0.003)$, and 166 potentially affected the positivity of weakly reactive samples (Figure 2). The O.D. values 167 slightly decreased with increased volume added $(150 \mu \mathrm{L}$ to $250 \mu \mathrm{L})$, but this difference was not 168 significant $(p=0.184)$ and did not affect positivity. In the non-reactive samples, the average O.D. 169 values of the flat-bottomed tubes $(0.080 \pm 0.008)$ were slightly higher than the conical tubes $170(0.059 \pm 0.021)$, but this difference was not significant $(p=0.323)$. Therefore, subsequent testing 171 was performed by eluting with $250 \mu \mathrm{L}$ PBS in flat-bottomed tubes to facilitate two tests per swab.

173 To determine the most efficient sample handling procedure to yield the highest O.D. values, 174 three methods were evaluated. In the reactive samples, there was little difference between the 175 O.D. values: undisturbed pellet $(0.688 \pm 0.434)$; supernatant $(0.545 \pm 0.222)$; resuspended pellet $176(0.531 \pm 0.214)$. One sample was a weak reactive in the pellet method (O.D. 0.258) but became 177 indeterminate in the supernatant (O.D. 0.214) and resuspended pellet (O.D. 0.197) method. 178 Pairwise comparison revealed no significant differences in O.D. values between the sample 
179 handing methods (pellet:supernatant, $\quad p=0.312 ; \quad$ pellet:resuspended, $\quad p=0.288$;

180 supernatant:resuspended, $p=0.515)$, but fractioning the sample could produce an indeterminate

181 result from a weakly reactive sample and was more labour intensive. Therefore, subsequent

182 testing was performed using the whole sample following centrifugation.

184 To determine the stability of oral swab collections at room temperature, O.D. values were

185 measured from dried oral swabs the day of collection and after two months of storage at room

186 temperature. The average O.D. value did not significantly differ between baseline (O.D. $0.575 \pm$

187 0.284) and two months (O.D. $0.568 \pm 0.188$ ). The mean difference in O.D. values was 0.008

188 ( $\mathrm{p}=0.946)$. No change is salivopositivity was observed.

\subsection{Diagnostic Accuracy Study}

191 To determine the correlation between oral flocked swabs, unstimulated saliva and serum a cross-

192 sectional diagnostic accuracy study was conducted on 50 volunteers for CMV, VZV, EBV

193 EBNA-1 and VCA, Measles, and Mumps IgG. For CMV IgG, the seropositivity using serum

194 was 24/50 (48.0\%). The cut-off O.D. values for swabs and saliva were non-reactive <0.179 and

195 reactive $>0.221$; and non-reactive $<0.220$ and reactive $>0.253$, respectively (Table 1 ). Using the

196 new cut-off O.D. values the sensitivity of swabs and saliva were 23/24 (95.8\%; 95\% CI: 78.1\%,

$197100 \%$ ) and 24/24 (100\%; 95\% CI: 83.7\%, 100\%), respectively (Table 2). Specificity of both

198 swabs and saliva were $100 \%$. One swab was indeterminate. The agreement beyond chance was

199 very good between oral swabs and both serum $(\mathrm{K}=0.88$; 95\% $\mathrm{CI}$ : 0.76, 1.000) and saliva 
$200(\mathrm{~K}=0.85 ; 95 \% \mathrm{CI}: 0.71,1.00)$, and perfect between saliva and serum $(\mathrm{K}=1.00 ; 95 \% \mathrm{CI}: 0.86$,

201 1.00) (Figure 3).

203 For VZV IgG, all participants were seropositive. There was excellent correlation between both 204 swabs and saliva to serum: 48/50 (96.0\%; 95\% CI: 85.7\%, 99.7\%) and 46/49 (93.9\%; 95\% CI: $82.9 \%, 98.5 \%)$, respectively. As there were no seronegative participants specificity could not be determined and Cohen's kappa coefficient was poor for both sera to swab and saliva $(\mathrm{K}=0)$, and

207 fair for swabs vs saliva $(\mathrm{K}=0.37 ; 95 \% \mathrm{CI}:-0.189,0.928)$.

210 and 44/50 (88.0\%), respectively. The sensitivity of swabs and saliva were comparable for both

211 EBNA-1 IgG [swabs: 35/38 (92.1\%; 95\% CI: 78.5\%, 98.0\%); saliva: 34/38 (91.9\%; 95\% CI:

$21275.3 \%, 96.4 \%)$ ] and VCA IgG [swabs: 42/44 (95.5\%; 95\% CI: 84.0\%, 99.6\%); saliva: 42/43

213 (97.7\%; 95\% CI: 86.8\%, 100\%)]. However, sensitivity for the composite measure of EBNA-1

214 IgG or VCA IgG equated to 43/44 (97.7\%; 95\% CI: 87.1\%, 100\%) and 44/44 (100\%; 95\% CI:

$21590.4 \%, 100 \%$ ) for swabs and saliva, respectively. The agreement for EBNA-1 IgG was very

216 good for both swab $(\mathrm{K}=0.85 ; 95 \% \mathrm{CI}: 0.68,1.00)$ and saliva $(\mathrm{K}=0.85 ; 95 \% \mathrm{CI}: 0.68,1.00)$

217 compared to serum, and perfect between swabs and saliva (K=1.000; 95\% CI: 0.90, 1.00). The

218 agreement for VCA IgG was very good for both swab (K=0.83; 95\% CI: 0.61, 1.000) and saliva

$219(\mathrm{~K}=0.91 ; 95 \% \mathrm{CI}: 0.74,1.00)$ compared to serum, and good between swabs and saliva $(\mathrm{K}=0.76$; $22095 \%$ CI: 0.50, 1.00). 
222 For Measles and Mumps IgG the correlation between oral swabs, unstimulated saliva and serum

223 as well as between two EIAs was determined. For Measles IgG, all participants were

224 seropositive by both assays. The sensitivity of swabs and saliva was much higher for the GSDx

225 assays for both swabs [41/48 (85.4\%; 95\% CI: 72.5\%, 93.1\%) vs 24/49 (48.9\%; 95\% CI: $35.6 \%$,

$22662.5 \%)$ and saliva [46/49 (93.9\%; 95\% CI: 82.9\%, 98.5\%) vs 34/49 (69.4\%; 95\% CI: 55.4\%,

$22780.6 \%)$ ]. However, the agreement for both assays between swab and saliva compared to sera

228 was poor $(\mathrm{K}=0)$, and poor for swab compared to saliva (GSDx: $\mathrm{K}=0.24 ; 95 \% \mathrm{CI}:-0.08,0.56$; $\mathrm{R}-$

229 Biopharm: $\mathrm{K}=0.27$; 95\% CI: -0.09, 0.46). Further analysis showed the R-Biopharm assay had a

230 much larger misclassification rate for both swab [25/50 (50.0\%; 95\% CI: 36.6\%, 63.4\%) vs 5/50

231 (10.0\%; 95\% CI: 3.9\%, 21.8\%)] and saliva [15/49 (30.6\%; 95\% CI: 19.5\%, 44.6\%) vs 3/49

$232(6.1 \%$; 95\% CI: 2.1\%, 16.5\%)], suggesting that the GSDx assay performs better for measuring

233 measles salivopositivity.

235 For Mumps IgG, both assays produced comparable seroprevalence of 45/50 (90.0\%) and 46/50

236 (92.0\%) for the GSDx and R-Biopharm assays, respectively. The sensitivity of both assays was

237 poor for both swabs [GSDx: 26/43 (60.5\%; 95\% CI: 45.6\%, 73.7\%); R-Biopharm: 28/45 (62.2\%;

238 95\% CI: 47.6\%, 74.9\%)] and saliva [GSDx: 30/44 (68.2\%; 95\% CI: 53.4\%, 80.1\%); R-

239 Biopharm: 33/45 (73.3\%; 95\% CI: 58.4\%, 84.2\%)]. ...(11). The agreement was only fair for

240 both assays between swab (GSDx: K=0.20; 95\% CI: 0.00, 0.40; R-Biopharm: K=0.21; 95\% CI:

$2410.03-0.40$ ) and saliva (GSDx: K=0.25; 95\% CI: 0.02, 0.47; R-Biopharm: K=0.32; 95\% CI: 0.07,

242 0.56) compared to sera, as well as swabs compared to saliva (GSDx: K=0.25; 95\% CI: 0.03,

2430.47 ; R-Biopharm: K=0.38; 95\% CI: 0.16, 0.60). Further analysis showed that both assays had a

244 large misclassification rate for both swab [GSDx: 18/44 (40.9\%; 95\% CI: 27.7\%, 55.6\%); R- 
245 Biopharm: $17 / 45$ (37.8\%; 95\% CI: 25.1\%, 52.4\%)] and saliva [GSDx: 15/45 (33.3\%; 95\% CI:

$24621.3 \%, 48.0 \%)$; R-Biopharm: 12/45 (26.7\%; 95\% CI: 15.8\%, 41.2\%)], suggesting that neither

247 assay is ideal for measuring mumps salivopositivity.

\section{4. Discussion}

249 Oral fluids contain IgG profiles highly similar to those in serum for a range of antigens and

250 diseases regardless of anatomical location (12). Their non-invasive collection is utilized in

251 several point-of-care assays to detect viral infections and immunity. To avoid degradation by

252 salivary endonucleases, diagnostic assays either utilize unadulterated gingival crevicular fluid or

253 whole saliva either chilled immediately following collection and stored frozen or stored in

254 biological stabilizers $(4,6)$. Studies for viral IgG utilize the Oracol saliva collection system

255 (Malvern Medical Developments, UK), which collects $1 \mathrm{~mL}$ saliva into $1 \mathrm{~mL}$ transport medium

256 allowing transportation at room temperature $(13,14)$. However, as samples must be stored

257 frozen for long-term preservation this is not ideal for field studies and resource-limited settings

258 (15). Therefore, as FLOQSwabs ${ }^{\circledR}$ are routinely used for bacteriology and molecular

259 microbiology $(16,17)$, we developed a simple pre-analytic and analytic method for detecting

260 viral sIgG on an open commercial platform using dried FLOQSwabs ${ }^{\circledR}$ that can be stored at room

261 temperature for up to 2-months. This was not an epidemiological study, but our method is

262 applicable to point-of-care or home testing, surveillance, clinical epidemiology and clinical

263 microbiology.

265 Our protocol was developed on a convenient cohort of healthy volunteers and appears promising

266 as swabs can be self-collected and diagnostic accuracy is comparable to saliva and serum for 
267 most viruses tested. The similarity between swabs and saliva is due to cell-free viral $\operatorname{IgG}$

268 secreted via the gingival crevicular fluid into the mouth and absorbed onto the swab regardless of

269 oral location swabbed. As swab absorption volume is much lower than the volume of saliva in

270 the mouth it is possible to collect multiple swabs consecutively at any time of day without a

271 reduction in positivity. Storing swabs dried maintains sample integrity and restricts activity of

272 salivary endonucleases for at least 2-months post-collection. Ideally, swabs could be collected

273 and shipped, but further investigation is needed to assess drying time on sample stability.

274 Nevertheless, a few procedural steps must be heeded. Whilst cut-off O.D. values vary between

275 assays, platforms and/or cohort tested, it is essential to elute swabs into flat-bottomed tubes to

276 prevent reabsorption and then test undiluted eluate. Elution with $250 \mu \mathrm{L}$ PBS permits each

277 sample to be tested twice. Further work is needed to (i) optimize elution and testing volumes to

278 maximize testing and increase O.D. values to resolve indeterminate samples, (ii) determine

279 sample integrity by comparing the absolute amount of human $\operatorname{IgG}$, and (iii) compare manual

280 testing vs automation to determine the robustness of the protocol.

282 Our swab protocol was initially developed for CMV due to interest from blood banks and

283 transplant programs. CMV is readily shed in saliva, the viral load is 100 -fold higher, and the

284 limit of detection is 10 -fold lower in saliva collected with a sterile swab than urine (18-20).

285 CMV antibody profiles in serum also strongly correlate with CMV infection and oral shedding

$286(8,21,22)$, and, based on our study, CMV IgG can be detected accurately in oral swabs. Swabs

287 displayed similar diagnostic accuracy to saliva and serum. The overall O.D. values in oral fluids

288 was lower than serum with swabs slightly lower than saliva but displayed a linear comparison in

289 O.D. values between serum and oral fluids. The slightly lower swab O.D. values compared to 
290 saliva results from a fraction of whole saliva diluted in eluate. The one swab that was equivocal

291 was strongly positive in sera and saliva suggesting further optimisation is warranted. Whilst our

292 protocol was developed for CMV IgG in healthy volunteers and may not be reflective of assay

293 performance in hospital or immunocompromised patient populations, it worked well for

294 herpesviruses due to maintained immunity from latent infections, but less than optimal for

295 measles and mumps possibly due to waning vaccine-induced immunity.

297 Few publications examined salivary IgG for VZV and EBV. In the only study on VZV IgG in

298 oral fluids, Talukder et al. examined 1,092 participants and reported a sensitivity and specificity

299 of $93 \%$ and $95.7 \%$, respectively (14). The sensitivity is comparable to saliva (93.9\%) and swabs

$300(96.0 \%)$ in our study, but our sample size was insufficient to determine assay specificity. In a

301 hospital laboratory volunteer study, virtually all employees have either received screening and

302 vaccination for vaccine-preventable illnesses or are old enough to have natural immunity. Based

303 on the diagnostic accuracy our procedure could be studied as a screening method for preschool

304 children susceptible to chicken pox.

306 Epidemiological screening for EBV, the aetiological agent of infectious mononucleosis and

307 nasopharyngeal carcinoma, is performed by screening for EBV VCA IgM, VCA IgG, and

308 EBNA-1 IgG via IFA or EIAs to distinguish acute from past infection (23). Both Vyse et al. and

309 Crowcroft et al. utilized oral fluids to determine EBV immune status $(24,25)$. Vyse et al. used a

310 ' $\mathrm{G}$ ' antibody capture radioimmunoassay for EBV VCA IgG and reported a sensitivity and

311 specificity of $93.5 \%$ and $100 \%$, respectively (25). Their assay was less sensitive than IFA as 
312 samples with a total $\mathrm{IgG}<2 \mathrm{mg} / \mathrm{L}$ yielded false positive reactions due to the monoclonal

313 antibody binding non-specifically to unsaturated anti-human IgG on the solid phase. Although

314 we didn't examine the total IgG in our samples, our protocol produced higher sensitivities for

315 both swabs and saliva with no known false positives. As 5\% of people do not produce EBNA-1

316 IgG after EBV infection we validated both EBV EBNA-1 IgG and VCA IgG (26). Combining

317 both assays our protocol yielded a sensitivity of $97.7 \%$ and $100 \%$ for swabs and saliva,

318 respectively, and may be useful for surveillance studies and could be preferred over serum

319 heterophile antibody testing for acute infectious mononucleosis work-up.

321 As Measles and Mumps are vaccine-preventable infections several studies have used oral fluids

322 as a non-invasive alternative for monitoring the efficacy of vaccination programs. For Measles

323 studies have used off-label either the commercialized Measles IgG Capture EIA (Microimmune

324 Ltd., UK), and the Enzygnost® Anti-Measles Virus/IgG (Siemens Health Care Diagnostics

$325 \mathrm{GmbH}$, Germany) with mixed results (5). Whilst Hayford et al., reported that oral fluids are not

326 suitable to detect immunity for Measles due to poor sensitivity (60.2\%) and specificity (75.7\%)

$327(27,28)$ others reported sensitivity of $90.0-92.0 \%$ and a specificity of $77.8-100 \%$ using the

328 Microimmune Ltd. assay (29-32). Our study also produced mixed results between the GSDx and

329 R-Biopharm assays. Whilst the R-Biopharm assay produced poorly, the GSDx assay was

330 comparable to the Microimmune Ltd. assay. Further optimization is required to increase the

331 sensitivity of our assay, but in its present stage may be adequate for epidemiological studies. If

332 the efficacy of vaccination is essential, confirmatory testing on serum should be performed for

333 non-reactive oral samples. 
335 For Mumps, Vainio et al., assessed the Mumps IgG Capture EIA (Microimmune Ltd.) and

336 reported low detection of Mumps IgG in oral samples (76\% sensitivity) and recommended that

337 the Microimmune assay not be used for surveillance studies (33). In our study, both the GSDx

338 and R-Biopharm assays produced similarly low sensitivities for both swabs and saliva, but

339 possibly for different reasons. The R-Biopharm assay produced low O.D. values for both oral

340 fluids and serum suggestive of an issue with the assay. The GSDx assay yielded a range of O.D.

341 values for serum similar in range to the other assays, but much lower $(<0.5)$ for swabs. Whilst it

342 is possible that Mumps IgG is not excreted into the mouth, vaccine-derived immunity to Mumps

343 also wanes especially into adulthood and maybe too low to detect in oral fluids (34).

345 Whilst the use of oral fluids is not novel, we developed a simple non-invasive protocol for

346 detecting viral IgG using oral flocked swabs. Based on the high sensitivity and excellent

347 correlation with serum, oral samples are ideal for CMV, EBV, and VZV, adequate for Measles,

348 but poor for Mumps. As samples can be stored dried for a few months, they are a viable option

349 for home and field collection. Future work should investigate the utility of oral swabs for viral

350 IgM as well as Rubella. Studies using oral fluids for Rubella IgM look promising with 79-96.9\%

351 sensitivity and 90-100\% specificity $(32,35)$. Nonetheless, oral swabs appear promising for

352 surveillance studies, transplant screening programs, and clinical microbiology. 


\section{5. CRediT Author Statement}

355 David J. Speicher: Conceptualization, Investigation, Writing - Original Draft; Kathy Luinstra:

356 Methodology, Writing - Review \& Editing; Emma J. Smith: Formal analysis; Santina

357 Castriciano: Conceptualization, Resources, Funding acquisition; Marek Smieja: Supervision

\section{Acknowledgements}

360 This study was supported in part by an award from The Research Institute of St. Joe's Hamilton.

361 We are grateful for the donation of FLOQSwabs ${ }^{\circledR}$ (\#520C) from Copan Italia, and commercial

362 EIA kits from Gold Standard Diagnostics and R-Biopharm AG. We thank Gold Standard

363 Diagnostics for lending the ThunderBolt ${ }^{\circledR}$ ELISA Analyzer. This study was based on the initial

364 CMV IgG testing by Dr Milena Furione and presented at the Clinical Virology Symposium

365 (CVS 2016; Poster \#230). Santina Castriciano is an employee of Copan Italia. The other authors

366 have no financial or other conflicts of interest to declare. Whilst this manuscript was shared with

367 Copan Italia and Gold Standard Diagnostics prior to submission these collaborators had no

368 influence on the data analysis or publication. We are thankful for the support from Jalees Nasir

369 for the statistical help. We are grateful to the many volunteers at St. Joseph's Healthcare

370 Hamilton who provided sera, saliva, and oral swabs for testing. 


\section{References}

372 1. Khan RS, Khurshid Z, Yahya Ibrahim Asiri F. 2017. Advancing Point-of-Care (PoC)

373 Testing Using Human Saliva as Liquid Biopsy. Diagnostics (Basel) 7.

374 2. Brandtzaeg P. 2007. Do salivary antibodies reliably reflect both mucosal and systemic 375 immunity? Ann N Y Acad Sci 1098:288-311.

376 3. Parry JV, Perry KR, Mortimer PP. 1987. Sensitive assays for viral antibodies in saliva: an 377 alternative to tests on serum. Lancet 2:72-5.

378 4. Saccoccio FM, Gallagher MK, Adler SP, McVoy MA. 2011. Neutralizing activity of 379 saliva against cytomegalovirus. Clin Vaccine Immunol 18:1536-42.

380 5. Dimech W, Mulders MN. 2016. A review of testing used in seroprevalence studies on $381 \quad$ measles and rubella. Vaccine 34:4119-4122.

382 6. Speicher DJ, Wanzala P, D'Lima M, Johnson KE, Johnson NW. 2015. Detecting DNA 383 viruses in oral fluids: evaluation of collection and storage methods. Diagn Microbiol $384 \quad$ Infect Dis 82:120-7.

385 7. Ljungman P, Hakki M, Boeckh M. 2011. Cytomegalovirus in hematopoietic stem cell 386 transplant recipients. Hematol Oncol Clin North Am 25:151-69.

387 8. Dollard SC, Keyserling H, Radford K, Amin MM, Stowell J, Winter J, Schmid DS, Cannon MJ, Hyde TB. 2014. Cytomegalovirus viral and antibody correlates in young children. BMC Res Notes 7:776.

390 9. Henson BS, Wong DT. 2010. Collection, storage, and processing of saliva samples for downstream molecular applications. Methods Mol Biol 666:21-30.

392 10. R Core Team. 2018. R: A language and environment for statistical computing., R 393 Foundation for Statistical Computing, Vienna, Austria. https://www.R-project.org/. 
394 11. Upper D. 1974. The unsuccessful self-treatment of a case of "writer's block". J Appl $395 \quad$ Behav Anal 7:497.

396 12. Hettegger P, Huber J, Passecker K, Soldo R, Kegler U, Nohammer C, Weinhausel A. 397 2019. High similarity of $\operatorname{IgG}$ antibody profiles in blood and saliva opens opportunities for saliva based serology. PLoS One 14:e0218456.

399 13. Furuta Y, Ohtani F, Aizawa H, Fukuda S, Kawabata H, Bergstrom T. 2005. Varicellazoster virus reactivation is an important cause of acute peripheral facial paralysis in children. Pediatr Infect Dis J 24:97-101.

402 14. Talukder Y, Gopal R, Andrews N, Glenn M, Breuer J, Brown D. 2005. Development and evaluation of Varicella zoster virus ELISA for oral fluid suitable for epidemiological studies. J Virol Methods 128:162-7.

405 15. Rosenbaum S, Gettler LT, McDade TW, Belarmino NM, Kuzawa CW. 2018. The effects of collection and storage conditions in the field on salivary testosterone, cortisol, and

408 16. Coorevits L, Vanscheeuwijck C, Traen A, Binge L, Ryckaert I, Padalko E. 2015. 409 Evaluation of Copan FLOQSwab for the molecular detection of Chlamydia trachomatis $410 \quad$ by Abbott RealTime CT PCR. Acta Clin Belg 70:398-402.

411 17. Larios OE, Coleman BL, Drews SJ, Mazzulli T, Borgundvaag B, Green K, Group ST-FS, McGeer AJ. 2011. Self-collected mid-turbinate swabs for the detection of respiratory viruses in adults with acute respiratory illnesses. PLoS One 6:e21335.

414 18. Cannon MJ, Stowell JD, Clark R, Dollard PR, Johnson D, Mask K, Stover C, Wu K, 415 Amin M, Hendley W, Guo J, Schmid DS, Dollard SC. 2014. Repeated measures study of 
weekly and daily cytomegalovirus shedding patterns in saliva and urine of healthy cytomegalovirus-seropositive children. BMC Infect Dis 14:569.

418 19. Barkai G, Ari-Even Roth D, Barzilai A, Tepperberg-Oikawa M, Mendelson E, Hildesheimer M, Kuint J. 2014. Universal neonatal cytomegalovirus screening using

421 20. Stowell JD, Mask K, Amin M, Clark R, Levis D, Hendley W, Lanzieri TM, Dollard SC, Cannon MJ. 2014. Cross-sectional study of cytomegalovirus shedding and immunological markers among seropositive children and their mothers. BMC Infect Dis

21. Cardoso ES, Jesus BL, Gomes LG, Sousa SM, Gadelha SR, Marin LJ. 2015. The use of saliva as a practical and feasible alternative to urine in large-scale screening for congenital cytomegalovirus infection increases inclusion and detection rates. Rev Soc

22. Wang JB, Adler SP. 1996. Salivary antibodies to cytomegalovirus (CMV) glycoprotein B accurately predict CMV infections among preschool children. J Clin Microbiol 34:2632-

432 23. De Paschale M, Clerici P. 2012. Serological diagnosis of Epstein-Barr virus infection: Problems and solutions. World J Virol 1:31-43.

434 24. Crowcroft NS, Vyse A, Brown DW, Strachan DP. 1998. Epidemiology of Epstein-Barr virus infection in pre-adolescent children: application of a new salivary method in Edinburgh, Scotland. J Epidemiol Community Health 52:101-4. 
437 25. Vyse AJ, Knowles WA, Cohen BJ, Brown DW. 1997. Detection of IgG antibody to

438 Epstein-Barr virus viral capsid antigen in saliva by antibody capture radioimmunoassay. J

$439 \quad$ Virol Methods 63:93-101.

440 26. De Paschale M, Agrappi C, Manco MT, Mirri P, Vigano EF, Clerici P. 2009.

441 Seroepidemiology of EBV and interpretation of the "isolated VCA IgG" pattern. J Med

$442 \quad$ Virol 81:325-31.

443 27. Hayford KT, Al-Emran HM, Moss WJ, Shomik MS, Bishai D, Levine OS. 2013.

444 Validation of an anti-measles virus-specific IgG assay with oral fluid samples for

445 immunization surveillance in Bangladesh. J Virol Methods 193:512-8.

446 28. Hayford KT, Shomik MS, Al-Emran HM, Moss WJ, Bishai D, Levine OS. 2013. Measles

447 vaccination coverage estimates from surveys, clinic records, and immune markers in oral

448 fluid and blood: a population-based cross-sectional study. BMC Public Health 13:1211.

449 29. Hutse V, Van Hecke K, De Bruyn R, Samu O, Lernout T, Muyembe JJ, Brochier B. 450 2010. Oral fluid for the serological and molecular diagnosis of measles. Int J Infect Dis $451 \quad 14: \mathrm{e} 991-7$.

452 30. Kremer JR, Muller CP. 2005. Evaluation of commercial assay detecting specific 453 immunoglobulin $\mathrm{g}$ in oral fluid for determining measles immunity in vaccinees. Clin 454 Diagn Lab Immunol 12:668-70.

455 31. Warrener L, Slibinskas R, Chua KB, Nigatu W, Brown KE, Sasnauskas K, Samuel D, 456 Brown D. 2011. A point-of-care test for measles diagnosis: detection of measles-specific 457 IgM antibodies and viral nucleic acid. Bull World Health Organ 89:675-82.

458 32. Nokes DJ, Enquselassie F, Nigatu W, Vyse AJ, Cohen BJ, Brown DW, Cutts FT. 2001. 459 Has oral fluid the potential to replace serum for the evaluation of population immunity 
levels? A study of measles, rubella and hepatitis B in rural Ethiopia. Bull World Health Organ 79:588-95.

462 33. Vainio K, Samdal HH, Anestad G, Wedege E, Skutlaberg DH, Bransdal KT, Mundal R, 463 Aaberge IS. 2008. Detection of measles- and mumps-specific IgG antibodies in paired 464 serum and oral fluid samples from Norwegian conscripts. Eur J Clin Microbiol Infect Dis $465 \quad 27: 461-5$.

466 34. Lewnard JA, Grad YH. 2018. Vaccine waning and mumps re-emergence in the United $467 \quad$ States. Sci Transl Med 10.

468 35. Vijaylakshmi P, Muthukkaruppan VR, Rajasundari A, Korukluoglu G, Nigatu W, 469 Warrener LA, Samuel D, Brown DW. 2006. Evaluation of a commercial rubella IgM 470 assay for use on oral fluid samples for diagnosis and surveillance of congenital rubella $471 \quad$ syndrome and postnatal rubella. J Clin Virol 37:265-8. 


\section{Figures}

474 Figure 1. Two-fold dilutions of the swab eluate in PBS with detection for CMV IgG. Dashed

475 lines indicate the reactive (upper; O.D. $=0.291$ ) and non-reactive (lower; O.D. $=0.222$ ) cut-off 476 O.D. values.

478 Figure 2. Graphical representation showing how flat-bottom (solid circles and line) and conical 479 (empty circles and dashed line) tube shape affects (A) volume recovered and (B) CMV sIgG 480 loads.

482 Figure 3. Comparison of viral-specific sIgG in paired flocked swab versus serum samples from 48350 healthy volunteers for the following targets: 1. CMV; 2. VZV; 3. EBV; 4. Measles; 5. 484 Mumps. For EBV both (a) EBNA-1 and (b) VCA were tested. For Measles and Mumps assays 485 from (a) Gold Standard Diagnostics and (b) R-Biopharm AG were investigated. Positive cut-off 486 values (dotted blue line) and negative cut-off values (dotted red line) were determined for each 487 assay. 


\section{$488 \quad$ Tables}

489 Table 1. Cut-off O.D. values for viral-specific IgG assays. For each assay the cut-off O.D.

490 values were determined as follows from the average O.D. values of all negative samples: Non-

491 reactive $=$ O.D. $<$ mean $($ non-reactive $)+2 \mathrm{St} . \mathrm{Dev} ;$ Indeterminate $=>$ mean $($ non-reactive $)+2 \mathrm{St}$

492 Dev but < mean (non-reactive) $+3 \mathrm{St} \mathrm{Dev}$; Reactive = O.D. $>$ mean (non-reactive) $+3 \mathrm{St} \mathrm{Dev}$.

\begin{tabular}{|l|l|c|c|c|c|c|c|}
\cline { 3 - 8 } \multicolumn{2}{c|}{} & \multicolumn{2}{c|}{ Serum } & \multicolumn{2}{c|}{ Flocked Swab } & \multicolumn{2}{c|}{ Saliva } \\
\hline \multicolumn{1}{|c|}{ Target } & Company & Non-Reactive & Reactive & Non-Reactive & Reactive & Non-Reactive & Reactive \\
\hline \hline CMV IgG & GSDx & 0.222 & 0.291 & 0.178 & 0.221 & 0.219 & 0.253 \\
\hline VZV IgG & R-Biopharm & 0.220 & 0.420 & 0.100 & 0.116 & 0.087 & 0.091 \\
\hline EBV EBNA-1 IgG & GSDx & 0.449 & 0.609 & 0.067 & 0.084 & 0.084 & 0.105 \\
\hline EBV VCA IgG & GSDx & 0.324 & 0.438 & 0.116 & 0.150 & 0.193 & 0.229 \\
\hline Measles IgG & R-Biopharm & 0.200 & 0.250 & 0.081 & 0.100 & 0.102 & 0.125 \\
\hline Measles IgG & GSDx & 0.275 & 0.400 & 0.101 & 0.116 & 0.115 & 0.135 \\
\hline Mumps IgG & R-Biopharm & 0.155 & 0.174 & 0.109 & 0.137 & 0.113 & 0.135 \\
\hline Mumps IgG & GSDx & 0.532 & 0.635 & 0.086 & 0.108 & 0.112 & 0.13 \\
\hline
\end{tabular}


495 Table 2. Diagnostic accuracy for viral-specific IgG assays in swabs and saliva compared to sera. For all samples were seropositive

496 for VZV and Measles so the specificity could not be determined.

\begin{tabular}{|c|c|c|c|c|c|c|c|c|c|c|c|c|c|c|c|c|}
\hline \multirow{3}{*}{$\begin{array}{l}\text { Assay } \\
\text { Specimen }\end{array}$} & \multirow{2}{*}{\multicolumn{2}{|c|}{$\begin{array}{c}\text { Cytomegalovirus } \\
\text { GSDx CMV IgG }\end{array}$}} & \multirow{2}{*}{\multicolumn{2}{|c|}{$\begin{array}{c}\begin{array}{c}\text { Varicella Zoster } \\
\text { Virus }\end{array} \\
\text { R-Bio VZV IgG } \\
\end{array}$}} & \multicolumn{4}{|c|}{ Epstein-Barr Virus } & \multicolumn{4}{|c|}{ Measles } & \multicolumn{4}{|c|}{ Mumps } \\
\hline & & & & & \multicolumn{2}{|c|}{ GSDx EBNA-1 IgG } & \multicolumn{2}{|c|}{ GSDx VCA IgG } & \multicolumn{2}{|c|}{ GSDx MeV IgG } & \multicolumn{2}{|c|}{ R-Bio MeV IgG } & \multicolumn{2}{|c|}{ GSDx MuV IgG } & \multicolumn{2}{|c|}{ R-Bio MuV IgG } \\
\hline & Swabs & Saliva & Swabs & Saliva & Swabs & Saliva & Swabs & Saliva & Swabs & Saliva & Swabs & Saliva & Swabs & Saliva & Swabs & Saliva \\
\hline Sample $(n=)$ & 50 & 49 & 50 & 49 & 50 & 49 & 50 & 499 & 50 & 49 & 50 & 49 & 50 & 49 & 50 & 49 \\
\hline Positives* & $23 / 24$ & $24 / 24$ & $48 / 50$ & $46 / 49$ & $35 / 38$ & $34 / 38$ & $42 / 44$ & $42 / 44$ & $41 / 50$ & $46 / 50$ & $24 / 50$ & $34 / 50$ & $27 / 45$ & $31 / 45$ & $28 / 46$ & $33 / 46$ \\
\hline Sensitivity & $95.8 \%$ & $100.0 \%$ & $96.0 \%$ & $93.9 \%$ & $92.1 \%$ & $91.9 \%$ & $95.5 \%$ & $97.7 \%$ & $85.4 \%$ & $93.9 \%$ & $49.0 \%$ & $69.4 \%$ & $60.5 \%$ & $68.2 \%$ & $62.2 \%$ & $73.3 \%$ \\
\hline Specificity & $100 \%$ & $100 \%$ & ND & ND & $100 \%$ & $100 \%$ & $100 \%$ & $100 \%$ & ND & ND & ND & ND & $80.0 \%$ & $80.0 \%$ & $100.0 \%$ & $100.0 \%$ \\
\hline PPV & $100 \%$ & $100 \%$ & $100 \%$ & $100 \%$ & $100 \%$ & $100 \%$ & $100 \%$ & $100 \%$ & $100 \%$ & $100 \%$ & $100 \%$ & $100 \%$ & $96.3 \%$ & $96.8 \%$ & $100.0 \%$ & $100.0 \%$ \\
\hline NPV & $96.3 \%$ & $100 \%$ & ND & ND & $80.0 \%$ & $80.0 \%$ & $75.0 \%$ & $85.7 \%$ & ND & ND & ND & ND & $19.0 \%$ & $22.2 \%$ & $19.0 \%$ & $25.0 \%$ \\
\hline Accuracy & $98.0 \%$ & $100 \%$ & $96.0 \%$ & $93.9 \%$ & $94.0 \%$ & $93.9 \%$ & $96.0 \%$ & $98.0 \%$ & $85.4 \%$ & $93.9 \%$ & $49.0 \%$ & $69.4 \%$ & $62.5 \%$ & $69.4 \%$ & $65.3 \%$ & $75.5 \%$ \\
\hline Misclassification & $4.0 \%$ & $0.0 \%$ & $4.0 \%$ & $6.1 \%$ & $6.0 \%$ & $6.1 \%$ & $4.0 \%$ & $2.0 \%$ & $10.0 \%$ & $6.1 \%$ & $50.0 \%$ & $30.6 \%$ & $40.9 \%$ & $33.3 \%$ & $37.8 \%$ & $26.7 \%$ \\
\hline Agreement (K) & 0.88 & 0.85 & 0.00 & 0.00 & 0.85 & 0.85 & 0.83 & 0.91 & 0.00 & 0.00 & 0.00 & 0.00 & 0.20 & 0.25 & 0.21 & 0.32 \\
\hline Linearity (Adj. $\mathrm{R}^{2}$ ) & 0.713 & 0.622 & 0.248 & 0.218 & 0.315 & 0.178 & 0.232 & 0.266 & 0.308 & 0.351 & 0.554 & 0.316 & 0.142 & 0.221 & 0.005 & 0.017 \\
\hline
\end{tabular}

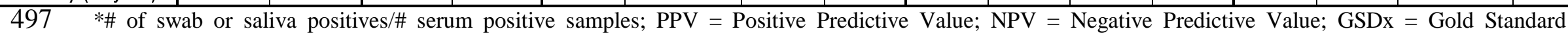

498 Diagnostics; R-Bio = R-Biopharm AG; ND = Not Determined. 


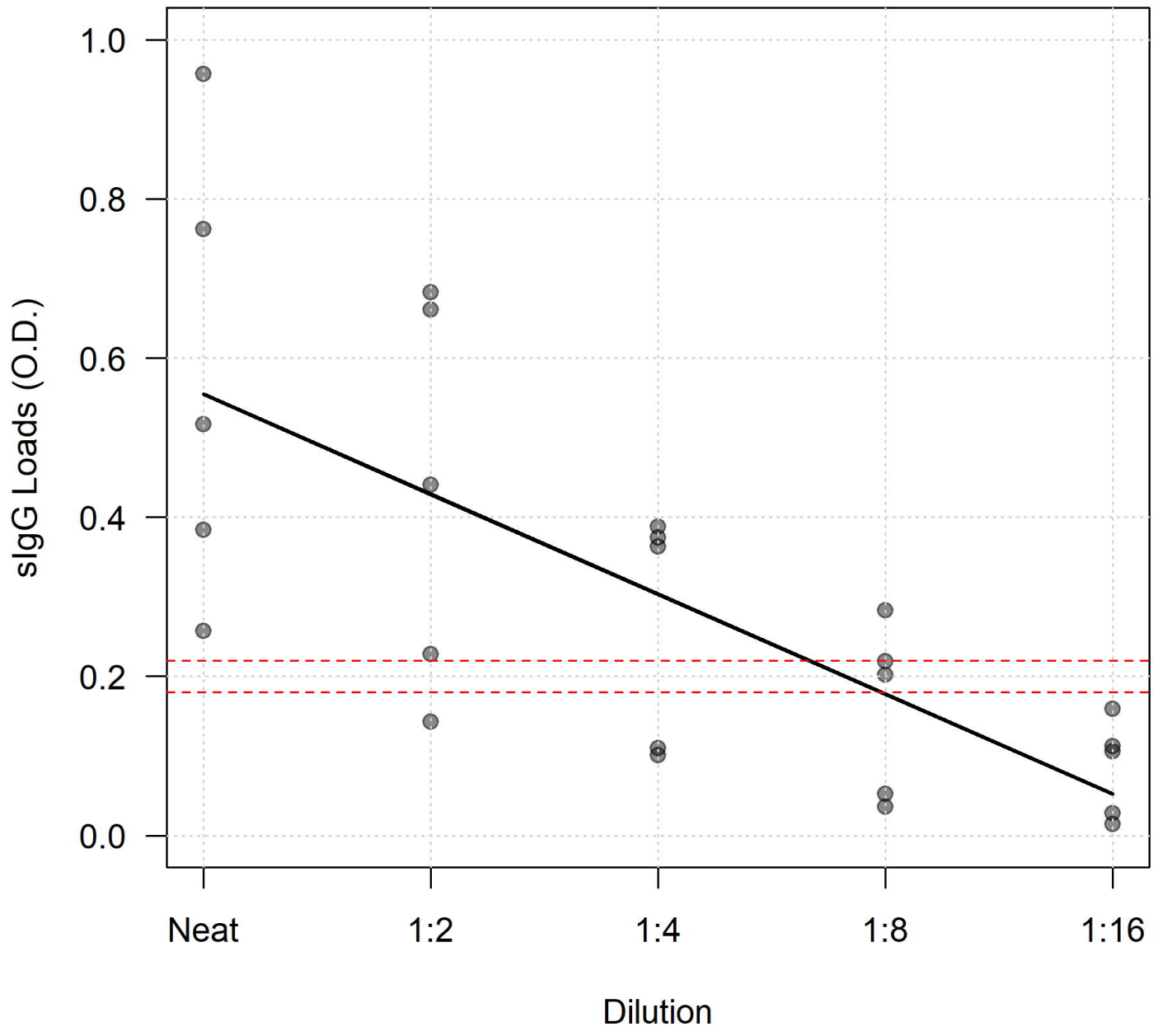




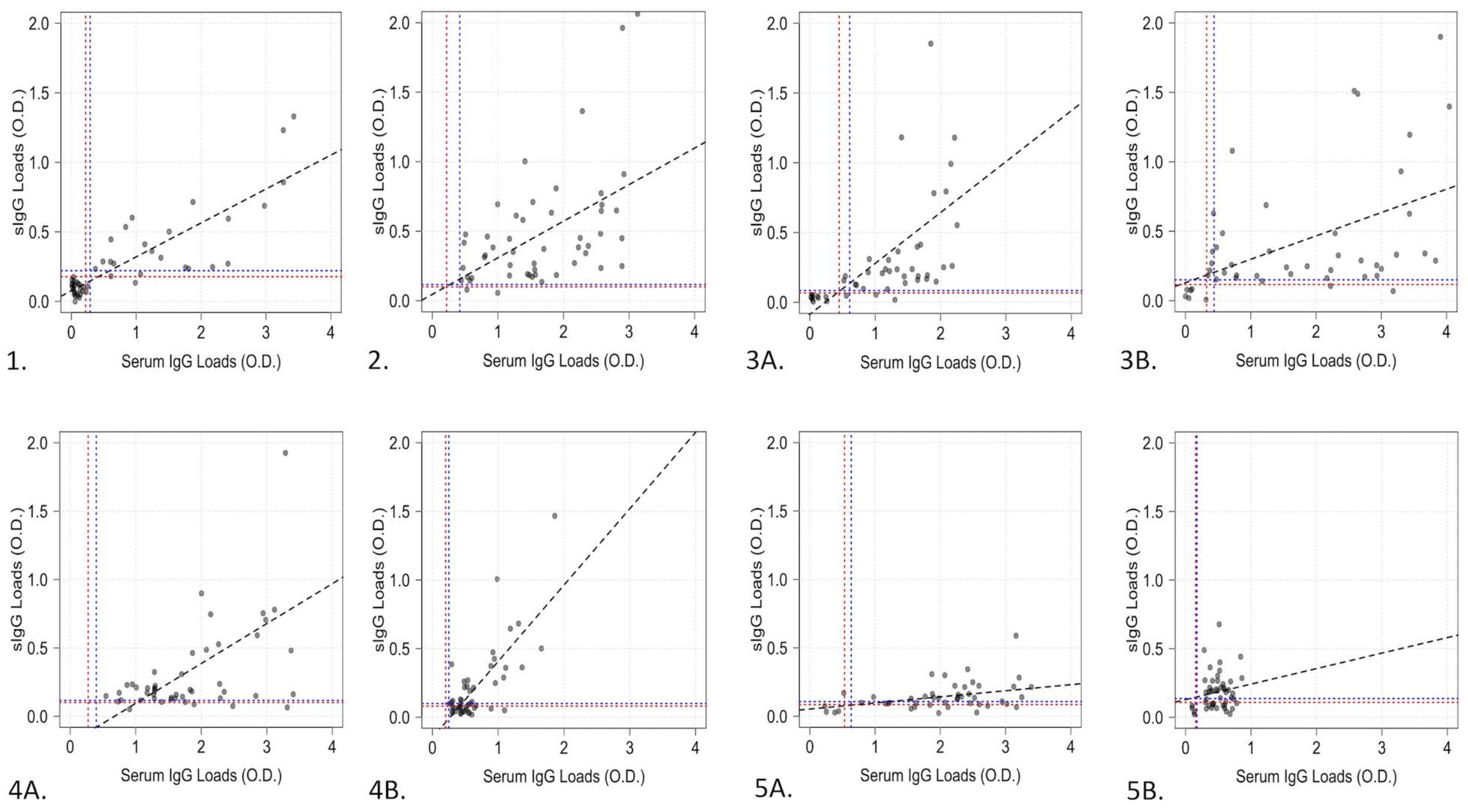

